\section{Routinely frozen biopsies of human skeletal muscle are suitable for morphological and immunocytochemical analyses at transmission electron microscopy}

M. Giagnacovo, ${ }^{1}$ R. Cardani, ${ }^{2,3}$ G. Meola, ${ }^{4}$

C. Pellicciari, ${ }^{1}$ M. Malatesta ${ }^{5}$

'Dipartimento di Biologia Animale, Laboratorio di Biologia cellulare e Neurobiologia, Università degli Studi di Pavia, Italy; '2Dipartimento di Biologia molecolare e Biotecnologie, Università degli Studi di Milano; ${ }^{3}$ Centro per lo studio delle Malattie Neuromuscolari CMN; ${ }^{4}$ Dipartimento di Neurologia, IRCCS Policlinico San Donato, Università degli Studi di Milano, Italy; ${ }^{5}$ Dipartimento di Scienze Neurologiche, Neuropsicologiche, Morfologiche e Motorie, Sezione di Anatomia e Istologia, Università degli Studi di Verona, Italy

\section{Abstract}

The aim of the present investigation was to evaluate whether routinely frozen biopsies of human skeletal muscle may be suitable for morphological and immunocytochemical analyses at transmission electron microscopy. The fixation/embedding protocols we successfully used for decades to process fresh mammalian tissues have been applied to frozen muscle biopsies stored for one to four years in liquid nitrogen. After $2.5 \%$ glutaraldehyde $2 \%$ paraformaldehyde - $1 \% \mathrm{OsO}_{4}$ fixation and embedding in epoxy resin, the ultrastructural morphology of myofibres and satellite cells as well as of their organelles and inclusions proved to be well preserved. As expected, after $4 \%$ paraformaldehyde - $0.5 \%$ glutaraldehyde fixation and embedding in LR White resin, the morphology of membrane-bounded organelles was relatively poor, although myofibrillar and sarcomeric organization was still recognizable. On the contrary, the myonuclei were excellently preserved and, after conventional staining with uranyl acetate, showed an EDTA-like effect, i.e. the bleaching of condensed chromatin, which allows the visualization of RNP-containing structures. These samples proved to be suitable for immunocytochemical analyses of both cytoskeletal and nuclear components, whereas the poor mitochondrial preservation makes unreliable any in situ investigation on these organelles.
Keeping in mind the limitations found, these results open promising perspectives in the study of frozen skeletal muscle samples stored in the tissue banks; this would be especially interesting for rare muscle diseases, where the limited number of biopsies suitable for ultrastructural investigation has so far represented a great restriction in elucidating the cellular mechanisms responsible for the pathological phenotype.

\section{Introduction}

Analysis by transmission electron microscopy (TEM) of human pathological biopsies is in general a difficult task, since the careful and immediate processing of the biological material is often hardly compatible with the surgical theatre.

After removal of the tissue from the body, spontaneous anoxic phenomena occur causing structural and molecular alterations: the shape, size and electron density of cell components may be modified by autolytic processes; some cellular materials (e.g. glycogen, lipids, lysosomal enzymes) may either disappear or re-locate; some enzyme activities may increase or decrease. ${ }^{1,2}$ This implies that TEM often represents a non-essential adjunct to light microscopy in pathological studies.

As a routine procedure, bioptic specimens are fixed in formalin to be processed for histological analysis at light microscopy, and/or frozen in liquid nitrogen and stored therein or at $-80^{\circ} \mathrm{C}$ for biochemical and histochemical studies.

Formalin-fixed samples have been already demonstrated to be suitable for some electron microscopy (EM) investigations, ${ }^{3 \cdot 7}$ but frozen specimens are frequently inadequate because of the ice crystals that form during the freezing process, thus damaging cellular components. ${ }^{8,9}$

Recently, a technique has been proposed to process frozen normal and pathologic pancreatic tissue for ultrastructural morphology; by this method, most of the histological and cytological features proved to be suitably preserved. ${ }^{10}$ However, scarce information exists on the possibility to use frozen biopsies of skeletal muscle for $\mathrm{EM}^{11}$ and in particular for ultrastructural immunocytochemistry.

In the present investigation, we applied to routinely frozen specimens of human skeletal muscle the fixation/embedding protocols successfully used for decades in our laboratories to process fresh mammalian tissues; the aim was to evaluate whether muscle biopsies stored for one to four years in liquid nitrogen can suitably be used for morphological and immunocytochemical analyses at TEM.
Correspondence: Manuela Malatesta, Dipartimento di Scienze Neurologiche, Neuropsicologiche, Morfologiche e Motorie, Sezione di Anatomia e Istologia, Università degli Studi di Verona, strada Le Grazie 8, 37134 Verona, Italy. E-mail:manuela.malatesta@univr.it

Key words: frozen biopsy, electron microscopy, fixation, immunocytochemistry, skeletal muscle.

Received for publication: 26 April 2010. Accepted for publication: 14 May 2010.

This work is licensed under a Creative Commons Attribution 3.0 License (by-nc 3.0).

○C Copyright M. Giagnacovo et al., 2010

Licensee PAGEPress, Italy

European Journal of Histochemistry 2010; $54: e 31$ doi:10.4081/ejh.2010.e31

\section{Materials and Methods}

\section{Tissue collection}

Samples of vastus lateralis and deltoideus muscles were taken from healthy donors, after informed consent. After removal, the biopsies were kept at $4^{\circ} \mathrm{C}$, trimmed of adipose and connective tissues, cut into fragments of approximately $5 \times 5 \times 5 \mathrm{~mm}$ in size and then immersed for $1 \mathrm{~min}$ in isopentane previously chilled with liquid nitrogen. The excess of isopentane was removed and the specimens were put in precooled $2 \mathrm{~mL}$ vials (Eppendorf, Hamburg, Germany), which were capped with a vented lid and stored in liquid nitrogen until further use. The frozen blocks used for the present study were stored for one to four years.

Other muscle tissue samples were cut into small fragments (about $1 \mathrm{~mm}^{3}$ ) immediately after removal from the body, and fixed by immersion following the same protocols used for frozen samples (see description below).

\section{Processing of samples for TEM}

The muscle samples were taken from storage in liquid nitrogen and immediately dipped into different fixative solutions previously cooled at $4^{\circ} \mathrm{C}$.

For conventional ultrastructural morphology, the specimens were fixed with $2.5 \%$ glutaraldehyde and $2 \%$ paraformaldehyde in $0.1 \mathrm{M}$ PBS, pH 7.4, for $2 \mathrm{~h}$ at $4^{\circ} \mathrm{C}$, rinsed with PBS, post-fixed with $1 \% \mathrm{OsO}_{4}$ for $2 \mathrm{~h}$ at room temperature, dehydrated with acetone and embedded in Epon 812. This procedure allows good morphological preservation, but most of the tissue molecules undergo denaturation, thus becoming undetectable by in situ cytochemical techniques. 
For immunoelectron microscopy, the samples were fixed with $4 \%$ paraformaldehyde and $0.5 \%$ glutaraldehyde in $0.1 \mathrm{M} \mathrm{PBS}$, pH 7.4, for 2 $\mathrm{h}$ at $4^{\circ} \mathrm{C}$. After washing in PBS, the specimens were treated with $0.5 \mathrm{M} \mathrm{NH}_{4} \mathrm{Cl}$ in PBS for 45 min at $4^{\circ} \mathrm{C}$ to block free aldehydes, dehydrated with graded concentrations of ethanol, and embedded in LR White resin. This procedure is optimal for antigen preservation, but the ultrastructural morphology is relatively poor, especially because of the lack of well-defined cellular membranes.

Attempting to improve the ultrastructural preservation of muscle components, further frozen samples were processed for both morphology and immunocytochemistry by using the same fixation solutions described above but containing $3 \mathrm{mM} \mathrm{CaCl}_{2}{ }^{1,2}$

Ultrathin sections (70-90 $\mathrm{nm}$ in thickness) of Epon-embedded samples were placed on copper grids and stained with uranyl acetate and lead citrate. Ultrathin sections of LR White-embedded samples were placed on nickel grids coated with a Formvar-carbon layer and then either stained as above or processed for immunocytochemistry.

\section{Ultrastructural immunocytochemistry}

To test the preservation of molecular reactivity in both the cytoplasm and nuclei of myofibres, some ultrathin sections were processed for immunocytochemistry using mouse monoclonal antibodies directed against the C-terminal end of actin (Clone AC-40, Sigma-Aldrich, Buchs, Switzerland; dilution 1:200), the heavy chain of skeletal fast fibre myosin (clone MY-32, Sigma-Aldrich; dilution $1: 200$ ), the phosphorylated form of RNA polymerase II (Research Diagnostic Inc., Flanders, NJ, USA; dilution 1:100), or the (Sm)snRNP (small nuclear ribonucleoprotein) core protein (Abcam, Cambridge, MA, USA; dilution 1:10). The sections were floated for $3 \mathrm{~min}$ on normal goat serum diluted 1:100 in PBS and then incubated for $17 \mathrm{~h}$ at $4^{\circ} \mathrm{C}$ with the primary antibody diluted in PBS containing $0.1 \%$ bovine serum albumin (Fluka, St. Louis, M0, USA) and $0.05 \%$ Tween 20 . After rinsing, sections were floated on normal goat serum, and then reacted for $30 \mathrm{~min}$ at room temperature with the appropriate secondary $12 \mathrm{~nm}$ gold-conjugated antibodies (Jackson Immuno Research Laboratories, West Grove, PA, USA) diluted 1:10 in PBS. The sections were rinsed, air-dried, and finally weakly stained with uranyl acetate. As controls, some grids were incubated without the primary antibody and then processed as described above.

All samples were observed in a Philips Morgagni transmission electron microscope equipped with a Megaview II camera for digital image acquisition.

\section{Results and Discussion}

In this study routinely frozen skeletal muscle biopsies were processed for TEM following the standard protocols used in our laboratories to process any kind of mammalian tissue samples. In our experience, these protocols gave excellent results when applied to skeletal muscle specimens fixed by immersion immediately after dissection. ${ }^{12-14}$

In the frozen muscle samples processed for conventional ultrastructural morphology (Figure 1), the general appearance of the tissue was almost comparable to muscle tissue immediately fixed after removing from the body (Figure 2), and no sign of ice-crystal damage was found. No difference was observed among samples kept in liquid nitrogen for different periods, thus indicating that the key fac- tor is the freezing process rather than the storage time. However, the ultrastructural morphology was found to be good in the outer part of the samples but it became relatively poor in the inner part (especially concerning mitochondrial morphology), so that the structural preservation may be considered as acceptable until about $2 \mathrm{~mm}$ depth.

The cytoplasm of myofibres was mostly occupied by longitudinally arrayed myofibrils, where the sarcomere architecture was clearly recognizable (Figure $1 \mathrm{a}, \mathrm{b}$ ) and the spatial arrangement of myofilaments was well preserved (inset in Figure 1b). Sarcomeres appeared to be more contracted in frozen samples than in immediately-fixed muscle tissue. This contraction is known to be due both to the biopsy procedure, which probably activates the muscle contractile mechanism, and to the slow action of the fixatives during fixation by
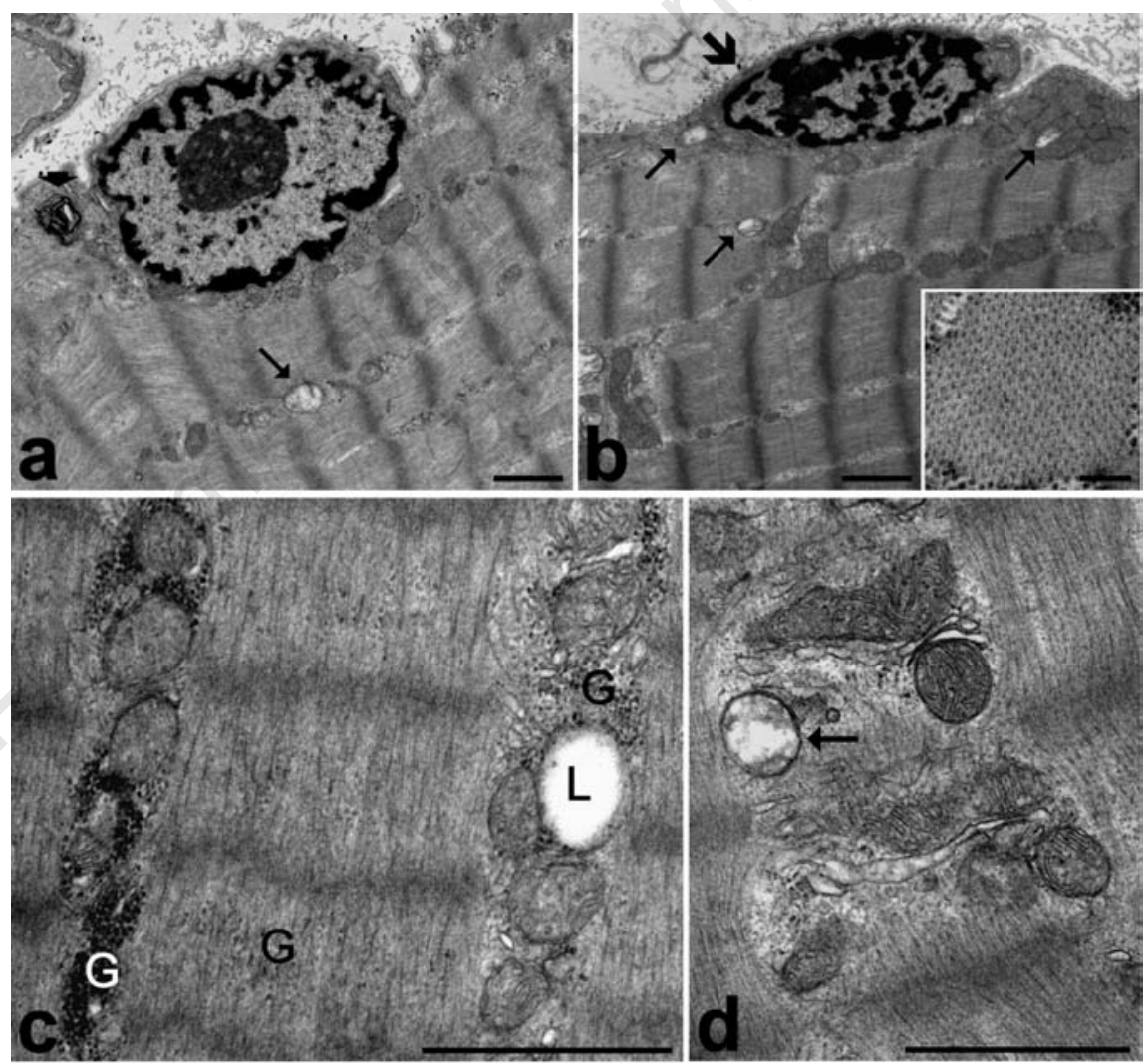

Figure 1. Frozen muscle biopsies stored in liquid nitrogen for one (c), three (d) or four (a, b) years, then fixed with $2.5 \%$ glutaraldehyde and $2 \%$ paraformaldehyde, post-fixed with $1 \% \mathrm{OsO}_{4}$ and embedded in Epon. a. The myofibre shows a well-preserved peripheral cell nucleus, cytoplasmic myofibrils longitudinally arranged in sarcomeres, and ovoid mitochondria lined along the myofibrils. Note a swollen damaged mitochondrion (arrow) and a myelin figure (arrowhead). b. A satellite cell (large arrow) showing a small heterochromatic nucleus. Arrows indicate swollen mitochondria (occurring both in the satellite cell and the adjacent myofibre) characterized by loss of cristae and a low matrix density. In the inset, a cross section of a myofibril is shown. c, d. Details of a myofibre cytoplasm. c. Glycogen (G) is present as both clusters and scattered among the myofibrils. A lipid droplet $(\mathrm{L})$ is partially surrounded by mitochondria. $d$. The membrane structures, as well as most of the mitochondria, are well preserved and show numerous cristae and electrondense matrix granules. Note the swollen mitochondrion devoid of cristae (arrow). Bars: $1 \mu \mathrm{m}$; inset $0.2 \mu \mathrm{m}$. 


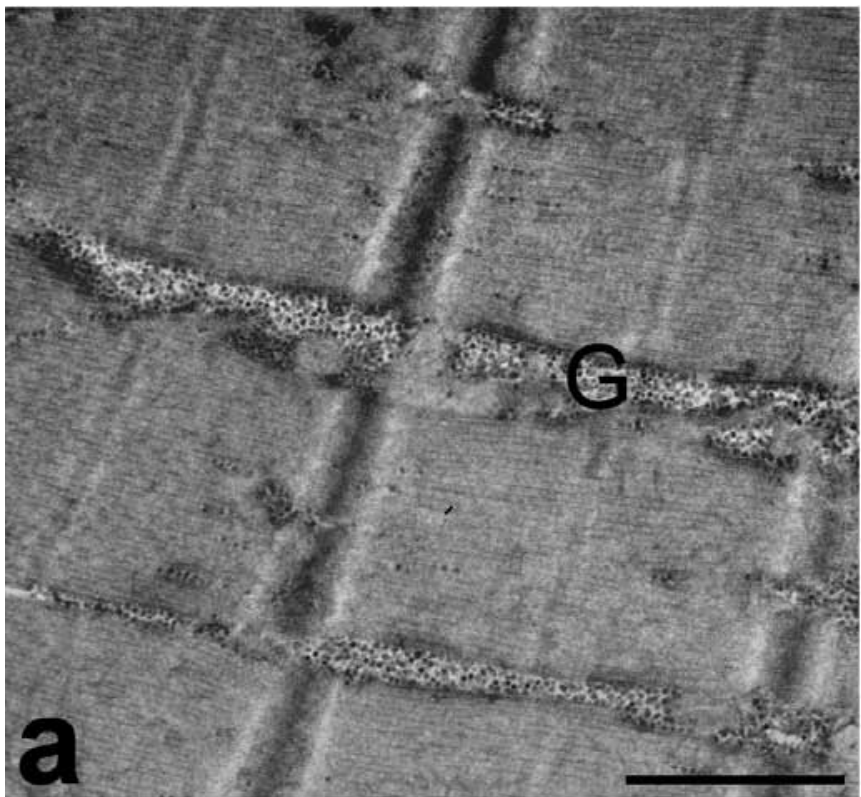

Figure 2. Muscle biopsies immediately fixed after removal from the body. a, b. Samples fixed with $2.5 \%$ glutaraldehyde and $2 \%$ paraformaldehyde, post-fixed with $1 \% \mathrm{OsO}_{4}$ and embedded in Epon. The cytoplasm shows well-preserved sarcomeres; glycogen (G) mostly occurs as clusters. Mitochondria (arrows) show numerous cristae and electron dense matrix granules, and frequently surround the lipid droplets (L). c. Samples fixed with $2 \%$ paraformaldehyde and $0.5 \%$ glutaraldehyde, and embedded in LR White. The sarcomeres are well visible and the mitochondria, which are lined between them, show still recognizable cristae (inset). Bars: a, c $1 \mu \mathrm{m}$; b, inset $0.5 \mu \mathrm{m}$.
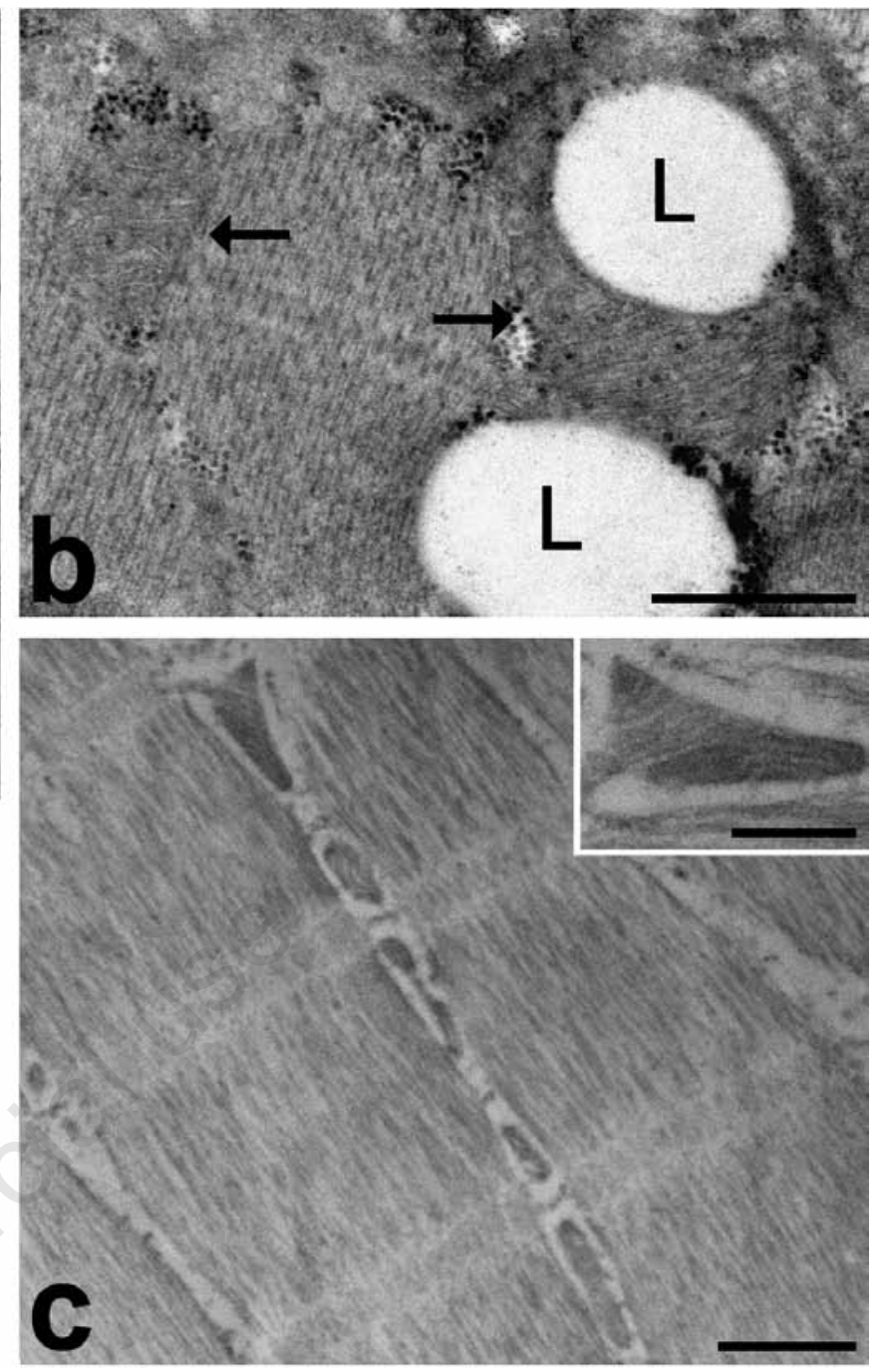

immersion (in fact, the contraction does not occur when fixation is performed by vascular perfusion). ${ }^{1,2}$ It is likely that the slower penetration of fixatives in the frozen samples may amplify this phenomenon, while the freezing process would not play a primary role in the induction of this artefact. The mitochondria were lined in small, longitudinally oriented cytoplasmic areas between the myofibrils (Figure 1a, b, c): the majority of mitochondria were ovoid-shaped with well preserved outer and inner membranes, numerous transverse cristae and a moderately electron dense matrix containing electron dense granules; only a few mitochondria showed rounded shapes, swelling, loss of cristae and lower matrix density (Figure 1a, b, d). The membrane structures were well preserved (Figure $1 \mathrm{~d}$ ), and no sign of the enlargement typical of post mortem alterations were ever found. Lipid droplets sometimes partially surrounded by mitochondria were properly maintained (Figure 1c). Glycogen was also preserved (Figure 1c), although it appeared more scattered in the cytoplasm compared to muscle samples imme- diately fixed after dissection. Glycogen is markedly affected by post mortem processes, and undergoes significant decrease, de-location and even disappearance, depending on the rapidity of fixative penetration; it is possible that, in the frozen samples, the penetration of aldehydes was rapid enough to prevent the complete loss of this cellular component but not its spreading. Some myelinic figures were occasionally observed in the cytoplasm (Figure 1a). Finally, cell nuclei occurred at the cell periphery, close to the plasma membrane: they generally showed finely irregular borders, small condensed chromatin clumps at both the nuclear and nucleolar periphery, and one or two roundish nucleoli (Figure 1a). No evidence of nuclear shape alteration or autolytic artefacts, such as massive clumping or condensation of chromatin, was ever found. Satellite cells were morphologically recognizable as small cells located between the sarcolemma and the surrounding basal lamina of the muscle fibres, and provided with scanty cytoplasm and one ovoid nucleus rich in condensed chromatin (Figure 1b). The ultrastruc- ture of the satellite cell components was well preserved, as in the myofibres.

Based on all these findings, the fixation/embedding protocols used for ultrastructural morphology proved to be suitable also for skeletal muscle samples submitted to routinary freezing and long-lasting storage in liquid nitrogen.

The crucial point for preserving ultrastructural morphology is the primary fixation; the subsequent post-fixation with $\mathrm{OsO}_{4}$ certainly improves the morphology by fixing lipid structures, and the gradual dehydration process minimizes possible volume changes of cell components, but they would be ineffective without a previous good fixation. It is known since the Sixties ${ }^{15}$ that fixatives containing both paraformaldehyde and glutaraldehyde give excellent preservation of a wide variety of tissues; paraformaldehyde penetrates tissues much more rapidly, and temporarily stabilises structures that are subsequently fixed more permanently by glutaraldehyde. Moreover, the addition of calcium ions to the fixative seems to stabilise the membranes probably by 
decreasing the extraction of lipids, ${ }^{2}$ although it is usually preferred to use low concentrations of divalent ions, since their excess may cause protein precipitation and excessive granularity. ${ }^{1}$ However, in this study the addition of $\mathrm{CaCl}_{2}$ did not improve the morphological features (not shown), the partial tissue damage observed being mainly due to the freezing procedure.

After fixation and processing for immunoelectron microscopy, the frozen muscle samples were characterised by a still recognizable myofibrillar and sarcomeric organization (Figure 3a), although this is less preserved than in samples immediately fixed after dissection (Figure 2c). The myonuclei were excellently preserved (Figure 3b) and, even at high magnification, no sign of the fine reticulation of nuclear content typical of ice crystal damage was observed. Interestingly, after conventional staining with uranyl acetate an EDTA-like effect was apparent, i.e. the bleaching of condensed chromatin, which allows the visualization of RNP structural components (perichromatin fibrils and granules, interchromatin granules, nuclear bodies). ${ }^{16}$ This phenomenon was observed to take place after embedding in some acrylic resins (e.g. Lowicryl K4M) or after cryofixation. ${ }^{17}$ On the other hand, mitochondria appeared roundish, shrunken and homogeneously electron dense (Figure 3c): this clearly indicates post mortem autolytic changes, which did not occur in bioptic muscle samples fixed immediately after removal from the body (Figure 2c). The fixative solution used for these samples was mainly composed of paraformaldehyde, which cannot give a good morphology but is necessary to maintain the immunocytochemical availability. Usually, glutaraldehyde is excluded from the fixation solutions for immunocytochemistry, since it brings in reactive molecular groups that could give rise to non-specific binding of the probes. In this study, it was added glutaraldehyde at low concentration $(0.5 \%)$ to improve organelle morphology in the frozen samples, and free aldehydes were blocked by using $\mathrm{NH}_{4} \mathrm{Cl}$; however, this precaution did not lead to fully satisfactory results. Similarly, the addition of $\mathrm{CaCl}_{2}$ to the fixative solution did not improve significantly the ultrastructural preservation of mitochondria, and only some of them showed hardly recognizable cristae (Figure $3 \mathrm{~d}$ ).

The results of the immunocytochemical detection of actin (Figure 4a) and myosin (Figure $4 \mathrm{~b}$ ) demonstrated a good retention of both the reactivity and intracellular distribution of these proteins. The anti-actin antibody gave a lower labelling compared to the antimyosin one; we cannot exclude that this may be due to the binding properties of the two probes used, but it is also worth reminding that actin is more affected than myosin by post
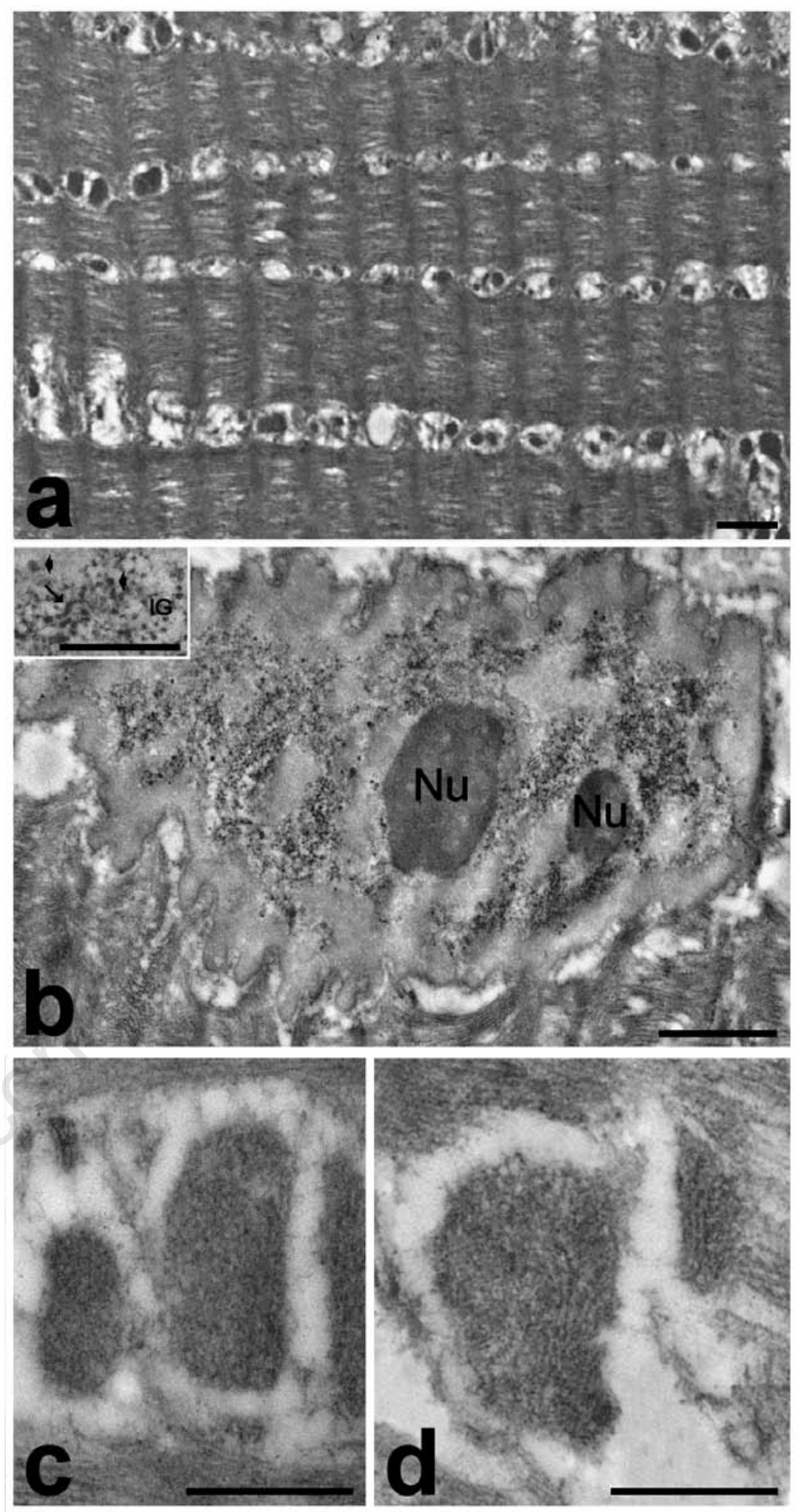

Figure 3. Frozen muscle biopsies stored in liquid nitrogen for three (a, c, d) or four (b) years, then fixed with $2 \%$ paraformaldehyde and $0.5 \%$ glutaraldehyde, and embedded in LR White. a. In the cytoplasm, the sarcomeric organization is still recognizable and mitochondria are arranged in longitudinal lines. b. Myonuclei show excellent ultrastructural preservation and an EDTA-like effect (bleaching of condensed chromatin revealing ribonucleoprotein structural constituents). In the inset perichromatin fibrils (arrow), perichromatin granules (arrowheads) and interchromatin granules (IG) are clearly visible. c. Mitochondria are shrunken and homogeneously electron dense, devoid of any sign of membranous structures. $d$. The addition of $\mathrm{CaCl}_{2}$ to the fixative solution did not significantly improve the ultrastructural preservation of mitochondria and only some mitochondria showed hardly recognizable cristae. Bars: a, b $1 \mu \mathrm{m}$; c, d, inset $0.5 \mu \mathrm{m}$. 
mortem proteolytic digestion. ${ }^{2}$ The immunolocalization of the nuclear factors is consistent with data in the literature: ${ }^{12-14}$ the anti-polymerase II labelling was mostly associated with perichromatin fibrils (i.e. the site of pre-mRNA transcription and early splicing ${ }^{18}$ ) (Figure 4c), while the anti-snRNP signal was restricted to perichromatin fibrils and interchromatin granules (i.e. storage/assembly site of splicing factors $^{18}$ ) (Figure 4d). All control samples showed negligible labelling.

The frozen samples are therefore suitable for immunocytochemical analyses on cytoskeletal and nuclear components, whereas the very poor mitochondrial preservation makes unreliable any in situ investigation on these organelles. However, it should be reminded that also Epon-embedded samples could be used for immunocytochemical studies by previously submitting ultrathin sections to an etching procedure with sodium metaperiodate or hydrogen peroxide, in order to improve antibody binding. ${ }^{19}$

In conclusion, this study demonstrates that routinely frozen biopsies of human skeletal muscle can be successfully processed for morphological and immunocytochemical studies at TEM by using the simple fixation-dehydrationembedding protocols usually applied to mammalian tissue specimens. It should be underlined that accurate procedures of biopsy freezing and storage are prerequisites for the appli- cation of the described methods.

Keeping in mind the limitations revealed by the present investigation, these results open promising perspectives for multiple exploitation of the bioptic muscle samples stored in tissue banks. This would be especially interesting for the ultrastructural immunolabeling of specific molecular markers of diagnostic value ${ }^{20}$, and in the study of rare muscle diseases (e.g., myotonic dystrophies $^{21-24}$ or laminopathies ${ }^{25-27}$ ), where the limited number of biopsies suitable for ultrastructural studies has so far represented a great restriction in investigating the cellular mechanisms responsible for the pathological phenotype.
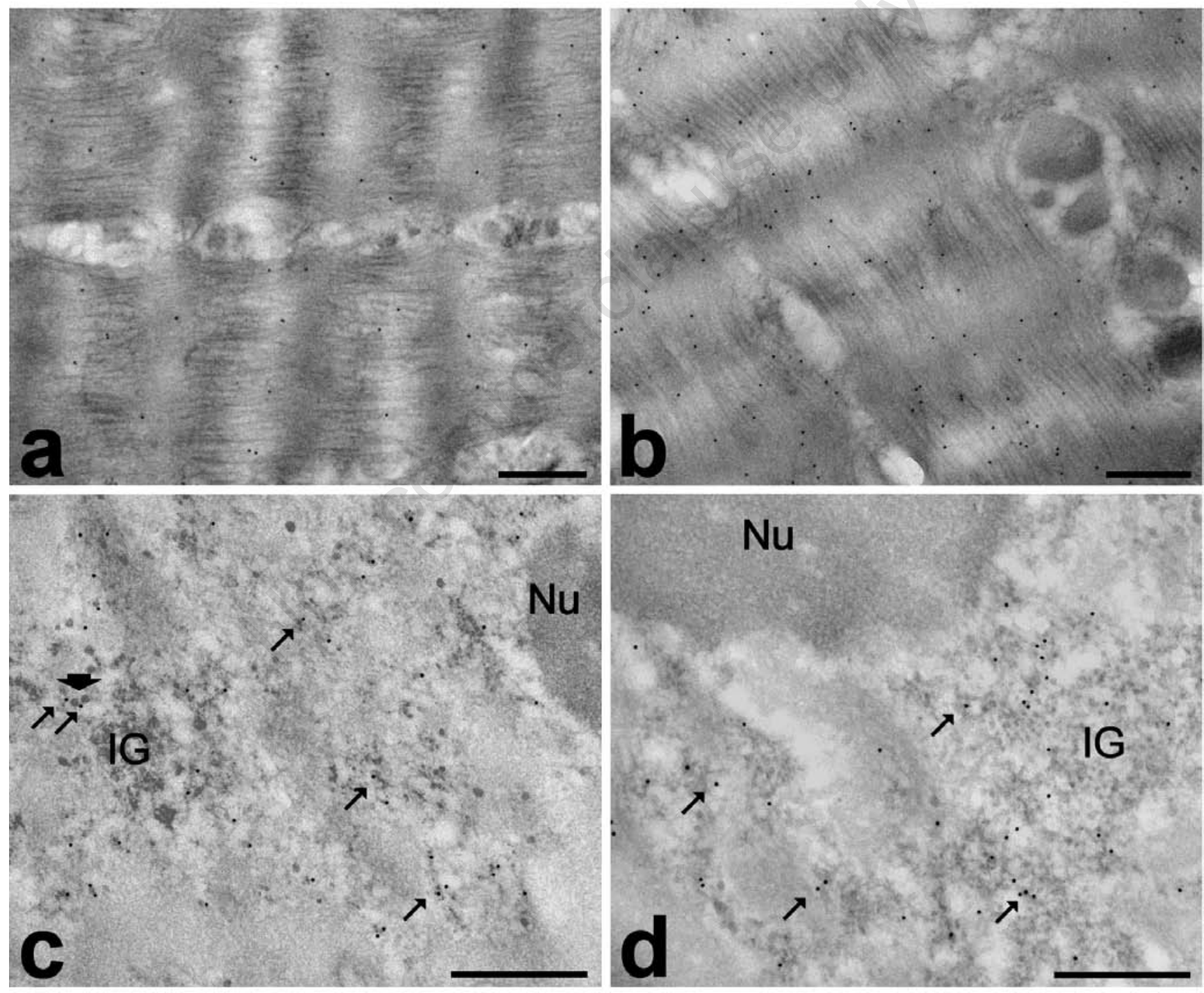

Figure 4. Frozen muscle biopsies stored in liquid nitrogen for one $(a, b)$ or four $(c, d)$ years, then fixed with $2 \%$ paraformaldehyde and $0.5 \%$ glutaraldehyde, embedded in LR White and processed for immunocytochemistry. Immunolabelling with anti-actin (a) and antimyosin (b): both probes bind specifically myofibrils. The anti-polymerase II antibody (c) labels perichromatin fibrils (arrows), even when they associate to perichromatin granules (arrowhead); anti-snRNP antibody (d) is associated with perichromatin fibrils (arrows) and interchromatin granules (IG). Nu: nucleolus. Bars: $0.5 \mu \mathrm{m}$. 


\section{References}

1. Glauert AM. Fixation, dehydration and embedding of biological specimens. Practical methods in electron microscopy. Ed. Glauert AM, North-Holland Publishing Company, Amsterdam - 0xford, and American Elsevier Publishing Company, New York, 1975.

2. Hayat MA. Principles and techniques of electron microscopy. Biological applications. Cambridge University Press, Cambridge, 2000.

3. Hultquist GT, Karlsson U. Use of formalinfixed, paraffin-embedded biopsy or autopsy material for electron microscopy. Pathol Eur 1972;7:97-101.

4. Wang NS, Minassian H. The formaldehyde-fixed and paraffin-embedded tissues for diagnostic transmission electron microscopy: a retrospective and prospective study. Hum Pathol 1987;18:715-27.

5. Cassella FP, Crosier S, Hay J. Needle-biopsy of paraffin blocks to obtain tissue for electron microscopy. Med Lab Sci 1989;46: 378-9.

6. Mount SL, Schwarz JE, Taatjes DJ. Prolonged storage of fixative for electron microscopy: effects on tissue preservation for diagnostic specimens. Ultrastruct Pathol 1997;21:195-200.

7. Lighezan R, Baderca F, Alexa A, Iacovliev M, Bonţe D, Murărescu ED et al. The value of the reprocessing method of paraffinembedded biopsies for transmission electron microscopy. Rom J Morphol Embryol 2009;50:613-7.

8. Sjöström M. Ice crystal growth in skeletal muscle fibres. J Microsc 1975;105:67-80.

9. Dalen H, Scheie P. A correlative study of the freezing patterns in rat myocardium using scanning and transmission electron microscopes. Acta Physiol Scand Suppl 1991;599:47-60.

10. Vogels IM, Hoeben KA, Van Noorden CJ. Rapid combined light and electron microscopy on large frozen biological samples. J Microsc 2009;235:252-8.

11. Anderson JR. Recommendations for the biopsy procedure and assessment of skeletal muscle biopsies. Virchows Arch 1997; 431:227-33.

12. Malatesta M, Perdoni F, Battistelli S, Muller S, Zancanaro C. The cell nuclei of skeletal muscle cells are transcriptionally active in hibernating edible dormice. BMC Cell Biol 2009;10:19.

13. Malatesta M, Perdoni F, Muller S, Pellicciari C, Zancanaro C. Pre-mRNA processing is partially impaired in satellite cell nuclei from aged muscles. J Biomed Biotechnol 2010:410-5.

14. Malatesta M, Perdoni F, Muller S, Zancanaro C, Pellicciari C. Nuclei of aged myofibres undergo structural and functional changes suggesting impairment in RNA processing. Eur J Histochem 2009; 53:97-106.

15. Karnowski MJ. A formaldehyde-glutaraldehyde fixative of high osmolarity for use in electron microscopy. J Cell Biol 1965; 27:137A-8A.

16. Bernhard W. A new staining procedure for electron microscopic cytology. J Ultrastruct Res 1969; 27:250-65.

17. von Schack ML, Fakan S, Villiger W. Some applications of cryosubstitution in ultrastructural studies of the cell nucleus. Biol Cell 1991;72:113-9.

18. Fakan S. Ultrastructural cytochemical analyses of nuclear functional architecture. Eur J Histochem 2004;48:5-14.

19. Bendayan M, Zollinger M. Ultrastructural localization of antigenic sites on osmium- fixed tissues applying the protein A-gold technique. J Histochem Cytochem 1983; 31:101-9.

20. Tews DS, Goebel HH. Diagnostic immunohistochemistry in neuromuscular disorders. Histopathology 2005;46:1-23.

21. Liquori CL, Ricker K, Moseley ML, Jacobsen JF, Kress W, Naylor SL, et al. Myotonic dystrophy type 2 caused by a CCTG expansion in intron 1 of ZNF9. Science 2001; 293:864-7.

22. Cardani R, Mancinelli E, Sansone V, Rotondo G, Meola G. Biomolecular identification of (CCTG)n mutation in myotonic dystrophy type 2 (DM2) by FISH on muscle biopsy. Eur J Histochem 2004; 48:437-42.

23. Cardani R, Mancinelli E, Rotondo G, Sansone V, Meola G. Muscleblind-like protein 1 nuclear sequestration is a molecular pathology marker of DM1 and DM2. Eur J Histochem 2006;50:177-82.

24 Cardani R, Mancinelli E, Giagnacovo M, Sansone V, Meola G. Ribonuclear inclusions as biomarker of myotonic dystrophy type 2, even in improperly frozen or defrozen skeletal muscle biopsies. Eur J Histochem 2009;53:107-12.

25. Maraldi NM, Mazzotti G, Rana R, Antonucci A, Di Primio R, Guidotti L. The nuclear envelope, human genetic diseases and ageing. Eur J Histochem 2007;51 Suppl 1:117-24.

26. Andrés V, González JM. Role of A-type lamins in signaling, transcription, and chromatin organization. J Cell Biol 2009; 187:945-57.

27. Marmiroli S, Bertacchini J, Beretti F, Cenni V, Guida M, De Pol A, et al. A-type lamins and signaling: the PI 3-kinase/Akt pathway moves forward. J Cell Physiol 2009;220:553-61. 